\title{
LOVÁSZ-SCHRIJVER SDP-OPERATOR, NEAR-PERFECT GRAPHS AND NEAR-BIPARTITE GRAPHS
}

\author{
S. BIANCHI, M. ESCALANTE, G. NASINI, L. TUNÇEL
}

\begin{abstract}
We study the Lovász-Schrijver lift-and-project operator $\left(\mathrm{LS}_{+}\right)$based on the cone of symmetric, positive semidefinite matrices, applied to the fractional stable set polytope of graphs. The problem of obtaining a combinatorial characterization of graphs for which the $\mathrm{LS}_{+}$-operator generates the stable set polytope in one step has been open since 1990. We call these graphs $\mathrm{LS}_{+}$-perfect. In the current contribution, we pursue a full combinatorial characterization of $\mathrm{LS}_{+}-$ perfect graphs and make progress towards such a characterization by establishing a new, close relationship among $\mathrm{LS}_{+}$-perfect graphs, near-bipartite graphs and a newly introduced concept of full-support-perfect graphs.
\end{abstract}

\section{INTRODUCTION}

The notion of a perfect graph was introduced by Berge in the early 1960s [5]. A graph is called perfect if each of its induced subgraphs has chromatic number equal to the size of a maximum cardinality clique in the subgraph. Perfect graphs have caught the attention of many researchers in the area and inspired numerous interesting contributions to the literature for the past fifty years. One of the main results in the seminal paper of Grötschel, Lovász and Schrijver [20] is that perfect graphs constitute a graph class where the Maximum Weight Stable Set Problem (MWSSP) can be solved in polynomial time. Some years later, the same authors proved a very beautiful, related result which connects a purely graph theoretic notion to polyhedrality of a typically nonlinear convex relaxation and to the integrality and equality of two fundamental polytopes:

Date: November 6, 2014.

Key words and phrases. stable set problem, lift-and-project methods, semidefinite programming, integer programming.

Some of the results in this paper were first announced in conference proceedings in abstracts [7, 8]. This research was supported in part by grants PID-CONICET 241, PICT-ANPCyT 0361, ONR N00014-12-10049, and a Discovery Grant from NSERC.

S. Bianchi: Universidad Nacional de Rosario, Argentina (e-mail: sbianchi@fceia.unr.edu.ar) M. Escalante: Universidad Nacional de Rosario, Argentina (e-mail: mariana@fceia.unr.edu.ar) G. Nasini: Corresponding author. Universidad Nacional de Rosario, Argentina (e-mail: nasini@fceia.unr.ac.ar) L. Tunçel: Department of Combinatorics and Optimization, Faculty of Mathematics, University of Waterloo, Waterloo, Ontario N2L 3G1, Canada (e-mail: ltuncel@uwaterloo.ca). 
Theorem 1. (Grötschel, Lovász and Schrijver [20, 21]) For every graph $G$, the following are equivalent:

(1) $G$ is perfect;

(2) $\operatorname{STAB}(G)=\operatorname{CLIQUE}(G)$;

(3) $\operatorname{TH}(G)=\operatorname{STAB}(G)$;

(4) $\operatorname{TH}(G)=\operatorname{CLIQUE}(G)$;

(5) $\mathrm{TH}(G)$ is polyhedral.

In the above theorem, $\operatorname{STAB}(G)$ is the stable set polytope of $G, \operatorname{CLIQUE}(G)$ is its clique relaxation and $\operatorname{TH}(G)$ is the theta body of $G$ defined by Lovász [27].

In the early 1990s, Lovász and Schrijver 28] introduced the semidefinite relaxation $\mathrm{LS}_{+}(G)$ of $\operatorname{STAB}(G)$ which is stronger than $\operatorname{TH}(G)$. Following the same line of reasoning used for perfect graphs, they proved that MWSSP can be solved in polynomial time for the class of graphs for which $\mathrm{LS}_{+}(G)=\operatorname{STAB}(G)$. We call these graphs $\mathrm{LS}_{+}$-perfect graphs [7]. The set of $\mathrm{LS}_{+}$-perfect graphs is known to contain many rich and interesting classes of graphs (e.g., perfect graphs, $t$-perfect graphs, wheels, anti-holes, near-bipartite graphs) and their clique sums. However, no combinatorial characterization of $\mathrm{LS}_{+}$-perfect graphs have been obtained so far.

There are many studies of various variants of lift-and-project operators applied to the relaxations of the stable set problem (see for instance, [34, 24, 15, 23, 9, 26, 16, 31, 22, 18, 19]). Why study $\mathrm{LS}_{+}$-perfect graphs? For example, if we want to characterize the largest family of graphs for which MWSSP can be solved in polynomial time, then perhaps, we should pick a tractable relaxation of $\operatorname{STAB}(G)$ that is as strong as possible. This reasoning would suggest that, we should focus on the strongest, tractable lift-and-project operator and reiterate it as much as possible while maintaining tractability of the underlying relaxation. Even though the (lower bound) analysis for the strongest lift-and-project operators is typically challenging, indeed, some work on the behaviour of the strongest lift-and-project operators applied to the stable set problem already exists (see [1] and the references therein). In the spectrum of strong lift-and-project operators which utilize positive semidefiniteness constraints, given the above results of Grötschel, Lovász and Schrijver, it seems clear to us that we should pick an operator that is at least as strong as $\operatorname{TH}(G)$. Among many of the convex relaxations that are closely related to $\mathrm{TH}(G)$ but stronger, $\mathrm{TH}(G)$ continues to emerge as the central object with quite special mathematical properties (see [10] and the references therein). Given that the operator $\mathrm{LS}_{+}(G)$ can be defined as the intersection of the matrix-space liftings of the odd-cycle polytope of $G$ and the theta body of $G$, by definition, $\operatorname{LS}_{+}(G)$ encodes and retains very interesting combinatorial information about the graph $G$. Then, the next question is why not focus on iterated (hence stronger) operator $\mathrm{LS}_{+}^{k}$ for $k \geq 2$ but small enough to maintain tractability? The answer to this is related to the above; but, it is a bit more subtle: in the lifted, matrix-space representation of $\mathrm{LS}_{+}$, if we remove the positive semidefiniteness constraint, we end up with the lifting of the operator LS (defined later). In this lifted matrix space, if we remove the restriction that the matrix be symmetric, we end up with the lifting of a weaker relaxation $\mathrm{LS}_{0}$. $\mathrm{LS}_{0}^{k}$ retains many interesting combinatorial properties of $G$, see [24, 25]. Moreover, Lovász and Schrijver proved that for every graph $G, \operatorname{LS}_{0}(G)=\operatorname{LS}(G)$. However, this property does not generalize to the 
iterated operators $\mathrm{LS}_{0}^{k}, \mathrm{LS}^{k}$, even for $k=2$, even if we require that the underlying graph $G$ be perfect (see [2, 3]). Therefore, $\mathrm{LS}_{+}$has many of the desired attributes for this purpose.

One of our main goals in this line of research is to obtain a characterization of $\mathrm{LS}_{+}$-perfect graphs similar to the one given in Theorem 1 for perfect graphs. More precisely, we would like to find an appropriate polyhedral relaxation of $\operatorname{STAB}(G)$ playing the role of $\operatorname{CLIQUE}(G)$ in Theorem 1, when we replace $\mathrm{TH}(G)$ by $\mathrm{LS}_{+}(G)$. In [7] we introduced the polyhedral relaxation $\operatorname{NB}(G)$ of $\operatorname{STAB}(G)$, which is, to the best of our knowledge, the tightest polyhedral relaxation of $\mathrm{LS}_{+}(G)$. Roughly speaking, $\mathrm{NB}(G)$ is defined by the family of facets of stable set polytopes of a family of graphs that are built from near-bipartite graphs by using simple operations so that the the stable set polytope of the resulting graph does not have any facets outside the class of facets which define the stable set polytope of near-bipartite graphs (for a precise definition of $\mathrm{NB}(G)$, see Section 2). In our quest to obtain the desired characterization of $\mathrm{LS}_{+}$-perfect graphs, $\mathrm{NB}(G)$ is our current best guess for replacing CLIQUE $(G)$ in Theorem 1. More specifically, we conjecture that the next four statements are equivalent.

Conjecture 2. For every graph $G$, the following four statements are equivalent:

(1) $\operatorname{STAB}(G)=\mathrm{NB}(G)$;

(2) $\operatorname{LS}_{+}(G)=\operatorname{STAB}(G)$;

(3) $\mathrm{LS}_{+}(G)=\mathrm{NB}(G)$;

(4) $\mathrm{LS}_{+}(G)$ is polyhedral.

Verifying the validity of Conjecture 2 is equivalent to determine the validity of the following two statements:

Conjecture 3. For every graph $G$, if $\operatorname{LS}_{+}(G)$ is polyhedral then $\operatorname{STAB}(G)=\operatorname{NB}(G)$.

Conjecture 4. For every graph $G$, if $\operatorname{LS}_{+}(G)=\operatorname{STAB}(G)$ then $\operatorname{STAB}(G)=\operatorname{NB}(G)$.

In [6], we made some progress towards proving Conjecture 3, by presenting an infinite family of graphs for which it holds. Recently, Conjecture 4 was verified for web graphs [17. In this contribution, we prove that Conjecture 4 holds for a class of graphs called fs-perfect graphs that stand for full-support-perfect graphs. This graph family was originally defined in [29] and includes the set of near-perfect graphs previously defined by Shepherd in [32].

One of the main difficulties in obtaining a good combinatorial characterization for $\mathrm{LS}_{+}$-perfect graphs is that the lift-and-project operator $\mathrm{LS}_{+}$(and many related operators) can behave sporadically under many well-studied graph-minor operations (see [16, 26]). Therefore, in the study of $\mathrm{LS}_{+}$-perfect graphs we are faced with the problem of constructing suitable graph operations and then deriving certain monotonicity or loose invariance properties under such graph operations. In this context, we present two operations which preserve $\mathrm{LS}_{+}-$imperfection in graphs.

In the next section, we begin with notation and preliminary results that will be used throughout the paper. We also state our main characterization conjecture on $\mathrm{LS}_{+}$-perfect graphs. In Section 3, we characterize fs-perfection in the family of graphs built from a minimally imperfect graph and one additional node. In Section 4 we prove the validity of the conjecture on fs-perfect graphs. In order to ease the reading of this contribution, the proofs of results on the $\mathrm{LS}_{+}$ 
operator are presented in Section 5. Section 6 is devoted to the conclusions and some further results.

\section{FURTHER DEFINITIONS AND PRELIMINARY RESUlTS}

2.1. Graphs and the stable set polytope. Throughout this work, $G$ stands for a simple graph with node set $V(G)$ and edge set $E(G)$. The complementary graph of $G$, denoted by $\bar{G}$, is such that $V(\bar{G})=V(G)$ and, for $E(\bar{G})=\{u v: u, v \in V(G), u v \notin E(G)\}$. For any positive integer $n, K_{n}, C_{n}$ and $P_{n}$ denote the graphs with $n$ nodes corresponding to a complete graph, a cycle and a path, respectively. We assume that in the cycle $C_{n}$ node $i$ is adjacent to node $i+1$ for $i \in\{1, \ldots, n-1\}$ and $n$ is adjacent to node 1 .

Given $V^{\prime} \subseteq V(G)$, we say that $G^{\prime}$ is a subgraph of $G$ induced by the nodes in $V^{\prime}$ if $V\left(G^{\prime}\right)=V^{\prime}$ and $E\left(G^{\prime}\right)=\left\{u v: u v \in E(G),\{u, v\} \subseteq V\left(G^{\prime}\right)\right\}$. When $V\left(G^{\prime}\right)$ is clear from the context we say that $G^{\prime}$ is a node induced subgraph of $G$ and write $G^{\prime} \subseteq G$. Given $U \subseteq V(G)$, we denote by $G-U$ the subgraph of $G$ induced by the nodes in $V(G) \backslash U$. For simplicity, we write $G-u$ instead of $G-\{u\}$. We say that $G_{E}$ is an edge subgraph of $G$ if $V\left(G_{E}\right)=V(G)$ and $E\left(G_{E}\right) \subseteq E(G)$.

Given the graph $G$, the set $\Gamma_{G}(v)$ is the neighbourhood of node $v \in V(G)$ and $\delta_{G}(v)=\left|\Gamma_{G}(v)\right|$. The set $\Gamma_{G}[v]=\Gamma_{G}(v) \cup\{v\}$ is the closed neighbourhood of node $v$. When the graph is clear from context we simply write $\Gamma(v)$ or $\Gamma[v]$. If $G^{\prime} \subseteq G$ and $v \in V(G), G^{\prime} \ominus v$ is the subgraph of $G$ induced by the nodes in $V\left(G^{\prime}\right) \backslash \Gamma[v]$. We say that $G^{\prime} \ominus v$ is obtained from $G^{\prime}$ by destruction of $v \in V(G)$.

A stable set in $G$ is a subset of mutually nonadjacent nodes in $G$ and a clique is a subset of pairwise adjacent nodes in $G$. The cardinality of a stable set of maximum cardinality in $G$ is denoted by $\alpha(G)$. The stable set polytope in $G, \operatorname{STAB}(G)$, is the convex hull of the characteristic vectors of stable sets in $G$. The support of a valid inequality of the stable set polytope of a graph $G$ is the subgraph induced by the nodes with nonzero coefficient in the inequality and a full-support inequality has $G$ as support.

If $G^{\prime} \subseteq G$ we can consider every point in $\operatorname{STAB}\left(G^{\prime}\right)$ as a point in $\operatorname{STAB}(G)$, although they do not belong to the same space (for the missing nodes, we take direct sums with the interval $[0,1]$, since originally $\left.\operatorname{STAB}(G) \subseteq \operatorname{STAB}\left(G^{\prime}\right) \oplus[0,1]^{V(G) \backslash V\left(G^{\prime}\right)}\right)$. Then, given any family of graphs $\mathcal{F}$ and a graph $G$, we denote by $\mathcal{F}(G)$ the relaxation of $\operatorname{STAB}(G)$ defined by

$$
\mathcal{F}(G)=\bigcap_{G^{\prime} \subseteq G ; G^{\prime} \in \mathcal{F}} \operatorname{STAB}\left(G^{\prime}\right) .
$$

If FRAC denotes the family of complete graphs of size two, following the definition (1), the polyhedron $\operatorname{FRAC}(G)$ is called the edge relaxation. It is known that $G$ is bipartite if and only if $\operatorname{STAB}(G)=\operatorname{FRAC}(G)$. Similarly, if CLIQUE denotes the family of complete graphs, $\operatorname{CLIQUE}(G)$ is the clique relaxation already mentioned and a graph is perfect if and only if $\operatorname{STAB}(G)=\operatorname{CLIQUE}(G)$ [14]. Moreover, if OC denotes the family of odd cycles, as a consequence of results in [28] we have the following

Remark 5. If $G-v$ is bipartite for some $v \in V(G)$ then $\operatorname{STAB}(G)=\operatorname{FRAC}(G) \cap \operatorname{OC}(G)$.

In 33. Shepherd defined a graph $G$ to be near-bipartite if $G \ominus v$ is bipartite for every $v \in V(G)$. We denote by NB the family of near-bipartite graphs. Since complete graphs and odd cycles are 
near-bipartite graphs, it is clear that

$$
\mathrm{NB}(G) \subseteq \operatorname{CLIQUE}(G) \cap \mathrm{OC}(G)
$$

2.2. Minimally imperfect, near-perfect and fs-perfect graphs. Minimally imperfect graphs are those graphs that are not perfect but after deleting any node they become perfect. The Strong Perfect Graph Theorem [13] (also see [11]; and see [12] for the related recognition problem) states that the only minimally imperfect graphs are the odd cycles and their complements.

Given a graph $G$ it is known that the full-rank constraint

$$
\sum_{u \in V} x_{u} \leq \alpha(G)
$$

is always valid for $\operatorname{STAB}(G)$. A graph is near-perfect if its stable set polytope is defined only by non-negativity constraints, clique constraints and the full-rank constraint [32]. Due to the results of Chvátal [14, near-perfect graphs define a superclass of perfect graphs and after [30] minimally imperfect graphs are also near-perfect. Moreover, every node induced subgraph of a near-perfect graph is near-perfect [32]. In addition, Shepherd [32] conjectured that near-perfect graphs could be characterized in terms of certain combinatorial parameters and established that the validity of the conjecture follows from the Strong Perfect Graph (then Conjecture, now Theorem). Therefore,

Theorem 6. ([13], [32]) A graph $G$ is near-perfect if and only if, for every $G^{\prime} \subseteq G$ minimally imperfect, the following two properties hold:

(1) $\alpha\left(G^{\prime}\right)=\alpha(G)$;

(2) for all $v \in V(G), \alpha\left(G^{\prime} \ominus v\right)=\alpha(G)-1$.

As a generalization of near-perfect graphs we consider the family of fs-perfect (full-support perfect) graphs. A graph is fs-perfect if its stable set polytope is defined only by non-negativity constraints, clique constraints and at most one single full-support inequality. Then, every node induced subgraph of an fs-perfect graph is fs-perfect. We say that a graph is properly fs-perfect if it is an imperfect fs-perfect graph. Clearly, near-perfect graphs are fs-perfect but we will see that fs-perfect graphs define a strict superclass of near-perfect graphs.

2.3. The $\mathbf{L S}_{+}$operator. In this section, we present the definition of the $\mathrm{LS}_{+}$-operator [28] and some of its well-known properties when it is applied to relaxations of the stable set polytope of a graph. In order to do so, we need some more notation and definitions.

We denote by $\mathbf{e}_{0}, \mathbf{e}_{1}, \ldots, \mathbf{e}_{n}$ the vectors of the canonical basis of $\mathbb{R}^{n+1}$ where the first coordinate is indexed zero. Given a convex set $K$ in $[0,1]^{n}$,

$$
\operatorname{cone}(K):=\left\{\left(\begin{array}{c}
x_{0} \\
x
\end{array}\right) \in \mathbb{R}^{n+1}: x=x_{0} y ; y \in K ; x_{0} \geq 0\right\} .
$$

Let $\mathbb{S}^{n}$ be the space of $n \times n$ symmetric matrices with real entries. If $Y \in \mathbb{S}^{n}, \operatorname{diag}(Y)$ denotes the vector whose $i$-th entry is $Y_{i i}$, for every $i \in\{1, \ldots, n\}$. Let 


$$
\begin{aligned}
M(K):=\left\{Y \in \mathbb{S}^{n+1}: \quad\right. & Y \mathbf{e}_{0}=\operatorname{diag}(Y), \\
& Y \mathbf{e}_{i} \in \operatorname{cone}(K), \\
& \left.Y\left(\mathbf{e}_{0}-\mathbf{e}_{i}\right) \in \operatorname{cone}(K), \forall i \in\{1, \ldots, n\}\right\} .
\end{aligned}
$$

Projecting this polyhedral lifting back to the space $\mathbb{R}^{n}$ results in

$$
\operatorname{LS}(K):=\left\{x \in[0,1]^{n}:\left(\begin{array}{l}
1 \\
x
\end{array}\right)=Y \mathbf{e}_{0}, \text { for some } Y \in M(K)\right\} .
$$

Clearly, $\operatorname{LS}(K)$ is a relaxation of the convex hull of integer solutions in $K$, i.e., $\operatorname{conv}\left(K \cap\{0,1\}^{n}\right)$.

Let $\mathbb{S}_{+}^{n}$ be the space of $n \times n$ symmetric positive semidefinite (PSD) matrices with real entries. Then

$$
M_{+}(K):=M(K) \cap \mathbb{S}_{+}^{n+1}
$$

yields the tighter relaxation

$$
\mathrm{LS}_{+}(K):=\left\{x \in[0,1]^{n}:\left(\begin{array}{l}
1 \\
x
\end{array}\right)=Y \mathbf{e}_{0}, \text { for some } Y \in M_{+}(K)\right\} .
$$

If we let $\operatorname{LS}^{0}(K):=K$, then the successive applications of the LS operator yield $\operatorname{LS}^{k}(K)=$ $\operatorname{LS}\left(\operatorname{LS}^{k-1}(K)\right)$ for every $k \geq 1$. Similarly for the $\mathrm{LS}_{+}$operator. Lovász and Schrijver proved that $\operatorname{LS}^{n}(K)=\operatorname{LS}_{+}^{n}(K)=\operatorname{conv}\left(K \cap\{0,1\}^{n}\right)$.

In this paper, we focus on the behaviour of the $\mathrm{LS}_{+}$operator on the edge relaxation of the stable set polytope. In order to simplify the notation we write $\mathrm{LS}_{+}(G)$ instead of $\mathrm{LS}_{+}(\mathrm{FRAC}(G))$ and similarly for the successive iterations of it. It is known [28] that, for every graph $G$,

$$
\operatorname{STAB}(G) \subseteq \mathrm{LS}_{+}(G) \subseteq \mathrm{TH}(G) \subseteq \operatorname{CLIQUE}(G)
$$

and

$$
\operatorname{STAB}(G) \subseteq \operatorname{LS}_{+}(G) \subseteq \mathrm{NB}(G) .
$$

2.4. $\mathbf{L S}_{+}$-perfect graphs. Recall that a graph $G$ is $\mathrm{LS}_{+}$-perfect if $\mathrm{LS}_{+}(G)=\operatorname{STAB}(G)$. A graph that is not $\mathrm{LS}_{+}$-perfect is called $\mathrm{LS}_{+}$-imperfect.

Using the results in [16] and [26] we know that all imperfect graphs with at most 6 nodes are $\mathrm{LS}_{+}$-perfect, except for the two properly near-perfect graphs depicted in Figure 1, denoted by $G_{L T}$ and $G_{E M N}$, respectively. These graphs prominently figure into our current work as the building blocks of an interesting family of graphs.

From the results in [28], it can be proved that every subgraph of an $\mathrm{LS}_{+}$-perfect graph is also $\mathrm{LS}_{+}$-perfect. Moreover, every graph for which $\operatorname{STAB}(G)=\mathrm{NB}(G)$ is $\mathrm{LS}_{+}$-perfect. In particular, perfect and near-bipartite graphs are $\mathrm{LS}_{+}$-perfect. Recall that in Conjecture 3 we wonder whether the only $\mathrm{LS}_{+}$-perfect graphs are those graphs $G$ for which $\operatorname{STAB}(G)=\mathrm{NB}(G)$. Obviously, $G$ is $\mathrm{LS}_{+}$-perfect if and only if every facet defining inequality of $\operatorname{STAB}(G)$ is valid for $\mathrm{LS}_{+}(G)$. In this context, we have the Lemma 1.5 in [28] that can be rewritten in the following way:

Theorem 7. Let ax $\leq \beta$ be a full-support valid inequality for $\operatorname{STAB}(G)$. If, for every $v \in V(G)$, $\sum_{w \in V(G-v)} a x \leq \beta-a_{v}$ is valid for $\operatorname{FRAC}(G \ominus v)$ then $a x \leq \beta$ is valid for $\operatorname{LS}_{+}(G)$. 

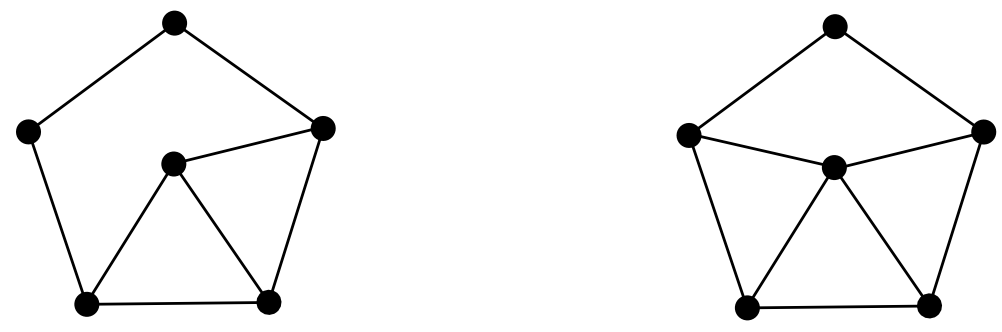

Figure 1. The graphs $G_{L T}$ and $G_{E M N}$.

In [6] we proved that the converse of the previous result is not true. However, it is plausible that the converse holds when the full-support valid inequality is a facet defining inequality of $\operatorname{STAB}(G)$. Actually, the latter assertion would be a consequence of the validity of Conjecture 3 . Thus, we present an equivalent formulation of it in the following.

Conjecture 8. If a graph is $\mathrm{LS}_{+}$-perfect and its stable set polytope has a full-support facet defining inequality, then the graph is near-bipartite.

2.5. Graph operations. In this section, we present some properties of four graphs operations that will be used throughout this paper. Firstly, let us recall the complete join of graphs. Given two graphs $G_{1}$ and $G_{2}$ such that $V\left(G_{1}\right) \cap V\left(G_{2}\right)=\emptyset$, we say that a graph $G$ is obtained after the complete join of $G_{1}$ and $G_{2}$, denoted $G=G_{1} \vee G_{2}$, if $V(G)=V\left(G_{1}\right) \cup V\left(G_{2}\right)$ and $E(G)=E\left(G_{1}\right) \cup E\left(G_{2}\right) \cup\left\{v w: v \in V\left(G_{1}\right)\right.$ and $\left.w \in V\left(G_{2}\right)\right\}$. A simple example of a join is the $n$-wheel $W_{n}$, for $n \geq 2$ which is the complete join of the trivial graph with one node and the $n$-cycle.

It is known that every facet defining inequality of $\operatorname{STAB}\left(G_{1} \vee G_{2}\right)$ can be obtained by the cartesian product of facets of $\operatorname{STAB}\left(G_{1}\right)$ and $\operatorname{STAB}\left(G_{2}\right)$. Hence, odd wheels are fs-perfect but not near-perfect graphs. Moreover, it is easy to see

Remark 9. The complete join of two graphs is properly fs-perfect if and only if one of them is a complete graph and the other one is a properly fs-perfect graph.

Also, it is known that

Remark 10. The complete join of two graphs is $\mathrm{LS}_{+}$-perfect if and only if both of them are $\mathrm{LS}_{+}$-perfect graphs.

Now, let us recall the odd-subdivision of an edge [35. Given a graph $G=(V, E)$ and $e \in E$, we say that the graph $G^{\prime}$ is obtained from $G$ after the odd-subdivision of the edge $e$ if it is replaced in $G$ by a path of odd length. Next, we consider the $k$-stretching of a node which is a generalization of the type (i) stretching operation defined in [26]. Let $v$ be a node of $G$ with neighborhood $\Gamma(v)$ and let $A_{1}$ and $A_{2}$ be nonempty subsets of $\Gamma(v)$ such that $A_{1} \cup A_{2}=\Gamma(v)$, and $A_{1} \cap A_{2}$ is a clique of size $k$. A $k$-stretching of the node $v$ is obtained as follows: remove $v$, introduce three nodes instead, called $v_{1}, v_{2}$ and $u$, and add an edge between $v_{i}$ and every node in $\{u\} \cup A_{i}$ for $i \in\{1,2\}$. Figure 2 illustrates the case $k=1$. 

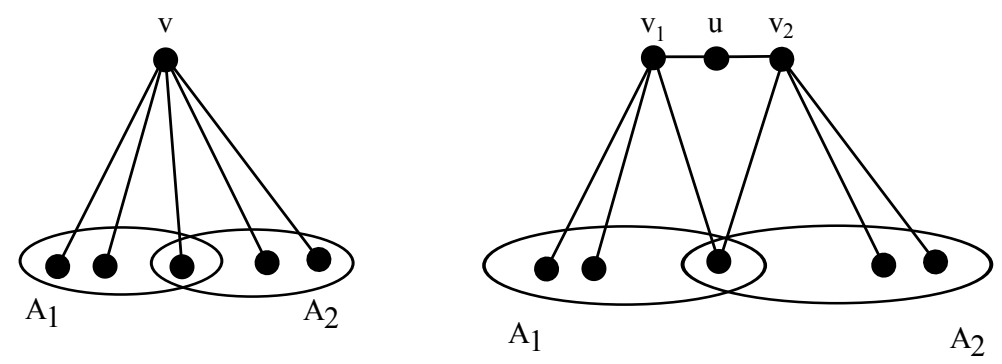

FiguRE 2. A 1-stretching operation on node $v$.
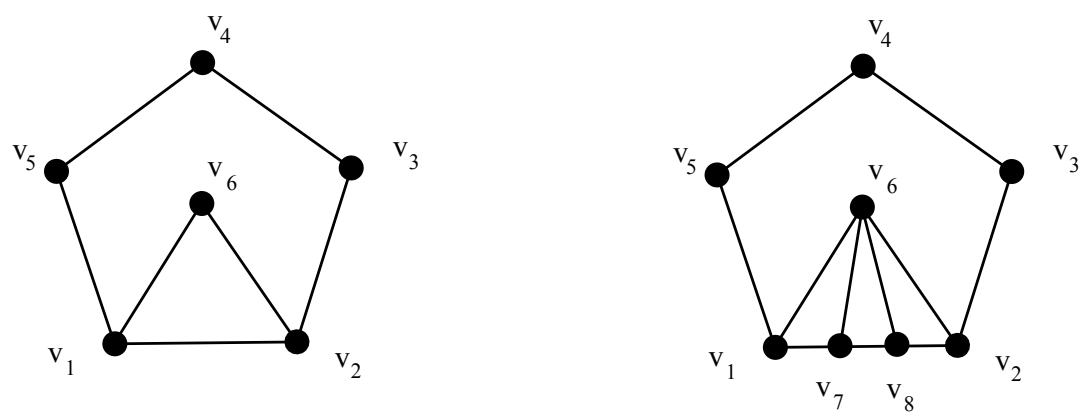

Figure 3. The graph $G^{\prime}$ is obtained from $G$ after the clique subdivision of edge $v_{1} v_{2}$.

The type (i) stretching operation presented in [26] corresponds to the case $k=0$.

We also consider another graph operation defined in [4]. Given the graph $G$ with nodes $\{1, \ldots, n\}$ and a clique $K=\left\{v_{1}, \ldots, v_{s}\right\}$ in $G$ (not necessarily maximal), the clique subdivision of the edge $v_{1} v_{2}$ in $K$ is defined as follows: delete the edge $v_{1} v_{2}$ from $G$, add the nodes $v_{n+1}$ and $v_{n+2}$ together with the edges $v_{1} v_{n+1}, v_{n+1} v_{n+2}, v_{n+2} v_{2}$ and $v_{n+i} v_{j}$ for $i \in\{1,2\}$ and $j \in\{3, \ldots, s\}$. Figure 3 illustrates the clique subdivision of the edge $v_{1} v_{2}$ in the clique $K=\left\{v_{1}, v_{2}, v_{3}\right\}$. Notice that if the clique is an edge this operation reduces to the odd-subdivision of it.

\section{3. fS-PERFECTION ON GRAPHS IN $\mathcal{F}^{k}$}

In order to prove Conjecture 8 on fs-perfect graphs, we first consider a minimal structure that a graph must have in order to be properly fs-perfect and $\mathrm{LS}_{+}$-imperfect. This leads us to define $\mathcal{F}^{k}$ for every $k \geq 2$ as the family of graphs having node set $\{0,1, \ldots, 2 k+1\}$ and such that $G-0$ is a minimally imperfect graph with $1 \leq \delta_{G}(0) \leq 2 k$. Let us consider necessary conditions for a graph in $\mathcal{F}^{k}$ to be fs-perfect.

Theorem 11. 29] Let $G \in \mathcal{F}^{k}$ be an fs-perfect graph. Then, the following conditions hold:

(1) $\alpha(G)=\alpha(G-0)$;

(2) $1 \leq \alpha(G \ominus 0) \leq \alpha(G)-1$;

(3) the full-support facet defining inequality of $\operatorname{STAB}(G)$ is the inequality

$$
(\alpha(G)-\alpha(G \ominus 0)) x_{0}+\sum_{i=1}^{2 k+1} x_{i} \leq \alpha(G) .
$$


Proof. Let

$$
\sum_{i=0}^{2 k+1} a_{i} x_{i} \leq \beta
$$

be the full-support facet defining inequality of $\operatorname{STAB}(G)$. We may assume that all coefficients $a_{i}, i \in\{0, \ldots, 2 k+1\}$ are positive integers. Clearly,

$$
\operatorname{STAB}(G-0)=\left\{x \in \operatorname{CLiQUE}(G-0): \sum_{i=1}^{2 k+1} a_{i} x_{i} \leq \beta\right\} \text {. }
$$

Since $G-0$ is a minimally imperfect graph, the inequality $\sum_{i=1}^{2 k+1} a_{i} x_{i} \leq \beta$ is a positive multiple of its rank constraint, i.e., there exists a positive integer number $p$ such that $a_{i}=p$ for $i \in$ $\{1, \ldots, 2 k+1\}$ and $\beta=p \alpha(G-0)$. Therefore, (2) has the form

$$
a_{0} x_{0}+p \sum_{i=1}^{2 k+1} x_{i} \leq p \alpha(G-0) \text {. }
$$

Observe that there is at least one root $\tilde{x}$ of $(3)$ such that $\tilde{x}_{0}=1$. Clearly, $\tilde{x}$ is the incidence vector of a stable set $S$ of $G$ such that $S-\{0\}$ is a maximum stable set of $G \ominus 0$. Then,

$$
a_{0}=p(\alpha(G-0)-\alpha(G \ominus 0)) .
$$

Since $a_{0} \geq 1$, we have $\alpha(G \ominus 0) \leq \alpha(G-0)-1$. Moreover, since $\delta_{G}(0) \leq 2 k$, then $\alpha(G \ominus 0) \geq 1$. Therefore, the inequality (3) becomes

$$
(\alpha(G-0)-\alpha(G \ominus 0)) x_{0}+\sum_{i=1}^{2 k+1} x_{i} \leq \alpha(G-0) .
$$

To complete the proof, we only need to show that $\alpha(G)=\alpha(G-0)$. Let $\bar{x}$ be the incidence vector of a maximum stable set in $G$, then

$$
\alpha(G)=\bar{x}_{0}+\sum_{i=1}^{2 k+1} \bar{x}_{i}
$$

Moreover, since $\alpha(G-0)-\alpha(G \ominus 0) \geq 1$ and $\bar{x}$ satisfies (4) we have

$$
\alpha(G)=\bar{x}_{0}+\sum_{i=1}^{2 k+1} \bar{x}_{i} \leq(\alpha(G-0)-\alpha(G \ominus 0)) \bar{x}_{0}+\sum_{i=1}^{2 k+1} \bar{x}_{i} \leq \alpha(G-0) .
$$

We have that $\alpha(G) \leq \alpha(G-0)$, implying $\alpha(G)=\alpha(G-0)$.

As a first consequence of the previous theorem we have:

Corollary 12. Let $G \in \mathcal{F}^{k}$ be such that $\alpha(G)=2$. Then, $G$ is fs-perfect if and only if $G-0=\overline{C_{2 k+1}}$ (the complementary graph of $C_{2 k+1}$ ) and $G$ is near-perfect.

Proof. Assume that $G$ is fs-perfect. By the previous theorem, $\alpha(G-0)=\alpha(G)=2$ and then $G-0=\overline{C_{2 k+1}}$. Moreover, $1 \leq \alpha(G \ominus 0) \leq \alpha(G)-1=1$ and $a_{0}=1$. Thus $G$ is near-perfect. The converse follows from the definition of fs-perfect graphs.

For $k \geq 2$, let $H^{k}$ denote the graph in $\mathcal{F}^{k}$ having $\alpha\left(H^{k}\right)=2$ and $\delta_{H^{k}}(0)=2 k$. Using Theorem 6 it is easy to check that $H^{k}$ is near-perfect. Using Corollary 12, we have the following result: 
Corollary 13. Let $G \in \mathcal{F}^{k}$ be such that $\alpha(G)=2$. Then, $G$ is fs-perfect if and only if $G$ is a near-perfect edge subgraph of $H^{k}$.

Let us now study the structure of fs-perfect graphs $G$ in $\mathcal{F}^{k}$ with stability number at least 3. From Theorem 11, $\alpha(G-0)=\alpha(G) \geq 3$ and $G-0=C_{2 k+1}$ with $k \geq 3$. Recall that in the cycle $C_{2 k+1}$ node $i$ is adjacent to node $i+1$ for $i \in\{1, \ldots, 2 k\}$ and $2 k+1$ is adjacent to node 1 . Clearly, if $\delta(0) \leq 2$ and $0 v \in E(G)$ then $G-v$ is bipartite and, by Remark 5 , $G$ is not fs-perfect.

From now on, $3 \leq s=\delta(0) \leq 2 k$ and $\Gamma(0)=\left\{v_{1}, \ldots, v_{s}\right\}$ such that $1 \leq v_{1}<\ldots<v_{s} \leq 2 k+1$. Observe that for every $i \in\{1, \ldots, s-1\}$, the nodes in $\left\{w \in V(G-0): v_{i} \leq w \leq v_{i+1}\right\}$ together with node 0 form a chordless cycle $D_{i}$ in $G$. Also, $D_{s}$ in $G$ is the chordless cycle induced by the nodes in $\left\{w \in V(G-0): v_{s} \leq w \leq 2 k+1\right.$ or $\left.1 \leq w \leq v_{1}\right\}$ and node 0 . We refer to these cycles as central cycles of $G$. It is easy to see, using parity arguments, that every $G \in \mathcal{F}^{k}$ has and odd number of odd central cycles. If $G$ has only one odd central cycle, say $D_{1}$, then $G-v_{1}$ is bipartite and, by Remark 5, $G$ is not fs-perfect.

We summarize the previous ideas in the following result:

Lemma 14. Let $G \in \mathcal{F}^{k}$ be a fs-perfect graph with $\alpha(G) \geq 3$. Then, $k \geq 3, G-0=C_{2 k+1}$ and $G$ has at least three odd central cycles.

According to lemma above, we can focus on structural properties of graphs in $\mathcal{F}^{k}$ with at least 3 odd central cycles. Firstly, we have:

Lemma 15. Let $G \in \mathcal{F}^{k}$ with $k \geq 3$ be such that $G-0=C_{2 k+1}$ and $G$ has at least 3 odd central cycles. Then, $G$ can be obtained after odd subdivisions of edges in a graph $G^{\prime} \in \mathcal{F}^{p}$ for some $2 \leq p<k$ with $\delta_{G^{\prime}}(0)=\delta_{G}(0)$. Moreover,

(1) if every central cycle of $G$ is odd, $G^{\prime}$ has one central cycle of length 5 and $2(p-1)$ central cycles of length 3 ;

(2) if $G$ has an even central cycle, every central cycle of $G^{\prime}$ has length 3 or 4.

Proof. Let $\delta_{G}(0)=s$ with $s \geq 3$.

(1) If every central cycle of $G$ is odd then $s$ is odd and $G$ has a central cycle with length at least 5. Let $p=\frac{s+1}{2}$ and $G^{\prime} \in \mathcal{F}^{p}$ with $\delta_{G^{\prime}}(0)=s$ such that 0 is adjacent to all nodes in $C_{2 p+1}$ except to two nodes, e.g., nodes $s$ and $s+1$. Clearly, $G^{\prime}$ has one 5 -central cycle and $s-1=2(p-1)$ central cycles of length 3 . Hence $G$ is obtained after the odd subdivision of edges of $G^{\prime}$.

(2) If $G$ has $r \geq 1$ even central cycles then $s+r$ is odd. Let $D_{i}$ with $i \in\{1, \ldots, s\}$ the central cycles of $G$. Let $p=\frac{s+r-1}{2}$ and $G^{\prime} \in \mathcal{F}^{p}$ such that $\delta_{G^{\prime}}(0)=s$ and for $i \in\{1, \ldots, s\}$ the central cycle $D_{i}^{\prime}$ in $G^{\prime}$ is:

(a) a 3 -cycle if $D_{i}$ is odd,

(b) a 4-cycle If $D_{i}$ is even.

It is straightforward to check that $G$ is obtained from $G^{\prime}$ after odd subdivision of edges.

In addition, we have 
Lemma 16. Let $G \in \mathcal{F}^{k}$ with $k \geq 3$ be such that $G-0=C_{2 k+1}$ and $\delta_{G}(0) \geq 3$. Let $t(G)$ be the number of 3-central cycles and $r(G)$ the number of 4-central cycles in $G$.

(1) if $G$ has three consecutive 3-central cycles then $G$ can be obtained after the clique subdivision of an edge in a graph $G^{\prime} \in \mathcal{F}^{k-1}$ with $t\left(G^{\prime}\right)=t(G)-2$ and $\delta_{G^{\prime}}(0)=\delta_{G}(0)-2$;

(2) if $r(G) \geq 2$ then $G$ can be obtained after the 1-stretching operation on a node in a graph $G^{\prime} \in \mathcal{F}^{k-1}$ with $r\left(G^{\prime}\right)=r(G)-1$ and $\delta_{G^{\prime}}(0)=\delta_{G}(0)-1$.

Proof. (1) From assumption we may consider that $\{2 k-1,2 k, 2 k+1,1\} \subseteq \Gamma(0)$. Let $G^{\prime} \in$ $\mathcal{F}^{k-1}$ be a graph having $\Gamma_{G^{\prime}}(0)=\Gamma(0) \backslash\{2 k, 2 k+1\}$. Clearly, $G^{\prime}$ has $t(G)-2$ 3-central cycles and $\delta_{G^{\prime}}(0)=\delta_{G}(0)-2$. Moreover, it is easy to see that $G$ is the clique subdivision of the edge in $G^{\prime}$ having extreme points $\{1,2 k-1\}$.

(2) Since there is a 4-central cycle, without loss of generality, we may assume that the nodes in $\{0,1,2 k, 2 k+1\}$ induce a 4 -central cycle in $G$. Consider $G^{\prime} \in F^{k-1}$ be such that $\Gamma_{G^{\prime}}(0)=\Gamma_{G}(0) \backslash\{2 k\}$ then $G$ is a 1 -stretching of $G^{\prime}$ performed at node 1 and where the new nodes are $2 k$ and $2 k+1$. Clearly, $r\left(G^{\prime}\right)=r(G)-1$ and $\delta_{G^{\prime}}(0)=\delta_{G}(0)-1$.

Utilizing the previous lemmas we obtain the following result.

Theorem 17. Let $G \in \mathcal{F}^{k}$ with $k \geq 3$ and $G$ has at least three odd central cycles. Then, $G$ can be obtained from $G_{L T}$ or $G_{E M N}$ after successively applying odd-subdivision of an edge, 1-stretching of a node and clique-subdivision of an edge operations.

Proof. Using Lemma 15, by successively performing the odd subdivision of an edge operation, we can restrict ourselves to consider the following cases:

(a) $G$ has one 5 -central cycle and $2(k-1) 3$-central cycles.

(b) $G$ has at least one even central cycle and every central cycle has length 3 or 4 .

Consider $r(G)$, the number of 4-cycles in $G$. If $r(G)=0$ then $G$ is a graph described in case (a). Since $k \geq 3$ then $2(k-1) \geq 4$ and by Lemma 16 (1), we can conclude that $G$ is obtained from $G_{L T}$ after successive clique-subdivisions of edges. For graphs in case (b) we have that $r(G) \geq 1$ and then by Lemma 15 (2) we have that $2 k=r(G)+\delta(G)-1$. If $r(G)=1$ and $\delta(G)$ is even, since $k \geq 3$ it holds that $G$ has $t(G)=\delta(G)-1 \geq 5$ number of 3-cycles. Using Lemma 16 (1) it is not hard to see that $G$ can be obtained from $G_{E M N}$ by successive clique subdivisions of edges. If $r(G) \geq 2$, Lemma 16 (2) implies that $G$ can be obtained by successive 1-stretching of nodes from a graph $G^{\prime} \in \mathcal{F}^{k^{\prime}}$ with $r\left(G^{\prime}\right)=1$ and $2 k^{\prime}=\delta\left(G^{\prime}\right)$. If $2 k^{\prime}=4$ then $G^{\prime}=G_{E M N}$ otherwise we can refer to the previous case and the proof is complete.

\section{The COnjeCture on fs-PERFECT GRAPhS}

In this section, we prove the validity of Conjecture 8 on the family of fs-perfect graphs. We start by proving it on graphs in the family $\mathcal{F}^{k}$ with $k \geq 2$. Recall that when $G \in \mathcal{F}^{k}$ is a fs-perfect graph with $\alpha(G)=2$, Corollary 13 states that $G$ is a near-perfect edge subgraph of $H^{k}$. Observe that $H^{2}=G_{E M N}$ and then, due to the results in [16], $H^{k}$ is $\mathrm{LS}_{+}$-imperfect when $k=2$. Next, we prove the imperfection property for the whole family of graphs $H^{k}$.

Theorem 18. For $k \geq 2$, the graph $H^{k}$ is $\mathrm{LS}_{+}$-imperfect. 
In order to ease the reading of this paper we postpone the proof of Theorem 18 to Section 5 . On the behaviour of the $\mathrm{LS}_{+}$operator on edge subgraphs, we have the following result:

Lemma 19. Let $G_{E}$ be an edge subgraph of $G$ and $a x \leq \beta$ be a valid inequality for $\operatorname{STAB}\left(G_{E}\right)$. Then, if ax $\leq \beta$ is not valid for $\operatorname{LS}_{+}^{r}(G)$, then $\operatorname{STAB}\left(G_{E}\right) \neq \operatorname{LS}_{+}^{r}\left(G_{E}\right)$.

Proof. Clearly, by definition $\operatorname{LS}_{+}^{r}(G) \subseteq \operatorname{LS}_{+}^{r}\left(G_{E}\right)$. Thus, if there exists $\hat{x} \in \operatorname{LS}_{+}^{r}(G)$ such that $a \hat{x}>\beta$ then $a x \leq \beta$ is not valid for $\operatorname{LS}_{+}^{r}\left(G_{E}\right)$. Moreover, by hypothesis, $a x \leq \beta$ is valid for $\operatorname{STAB}\left(G_{E}\right)$ and the result follows.

As a consequence of the above, we have:

Theorem 20. Let $G \in \mathcal{F}^{k}$ be a fs-perfect graph with $\alpha(G)=2$. Then, $G$ is $\mathrm{LS}_{+}$-imperfect.

Proof. By Corollary 13 we know that $G$ is a near-perfect edge subgraph of $H^{k}$. Theorem 18 states that $H^{k}$ is $\mathrm{LS}_{+}$-imperfect then the full rank constraint is not valid for $\operatorname{LS}_{+}\left(H^{k}\right)$. Since $\alpha\left(H^{k}\right)=\alpha(G)=2$, the full rank constraint is not valid for $\mathrm{LS}_{+}(G)$ and $G$ is $\mathrm{LS}_{+}$-imperfect.

Let us consider the fs-perfect graphs $G$ in $\mathcal{F}^{k}$ with $\alpha(G) \geq 3$. Due to the structural characterization in Theorem 17, we are interested in the behavior of the $\mathrm{LS}_{+}$-operator under the odd subdivision of an edge, $k$-stretching of a node and clique subdivision of an edge operations. In this context, a related earlier result is:

Theorem 21 ([26]). Let $G$ be a graph and $r \geq 1$ such that $\operatorname{LS}_{+}^{r}(G) \neq \operatorname{STAB}(G)$. Further assume that $\tilde{G}$ is obtained from $G$ by using the odd subdivision operation on one of its edges. Then, $\operatorname{LS}_{+}^{r}(\tilde{G}) \neq \operatorname{STAB}(\tilde{G})$.

Concerning the remaining operations, we present the following results whose proofs are included in Section 5 for the sake of clarity.

Theorem 22. Let $G$ be a graph and $r \geq 1$ such that $\operatorname{LS}_{+}^{r}(G) \neq \operatorname{STAB}(G)$. Further assume that $\tilde{G}$ is obtained from $G$ by using the $k$-stretching operation on one of its nodes. Then, $\operatorname{LS}_{+}^{r}(\tilde{G}) \neq$ $\operatorname{STAB}(\tilde{G})$.

Theorem 23. Let $G$ be a graph and $r \geq 1$ such that $\operatorname{LS}_{+}^{r}(G) \neq \operatorname{STAB}(G)$. Further assume that $\tilde{G}$ is obtained from $G$ by using the clique subdivision operation on one of its edges. Then, $\operatorname{LS}_{+}^{r}(\tilde{G}) \neq \operatorname{STAB}(\tilde{G})$.

In summary, we can conclude that the odd-subdivision of an edge, the 1-stretching of a node and the clique-subdivision of an edge are operations that preserve $\mathrm{LS}_{+}$-imperfection. Then, the behavior of these operations under the $\mathrm{LS}_{+}$operator together with the fact that graphs $G_{L T}$ and $G_{E M N}$ are $\mathrm{LS}_{+}$-imperfect, Lemma 14 and Theorem 17 allow us to deduce:

Theorem 24. Let $G \in \mathcal{F}^{k}$ be a fs-perfect graph with $\alpha(G) \geq 3$. Then, $G$ is $\mathrm{LS}_{+}$-imperfect.

Finally, we are able to present the main result of this contribution.

Theorem 25. Let $G$ be a properly fs-perfect graph which is also $\mathrm{LS}_{+}$-perfect. Then, $G$ is the complete join of a complete graph (possibly empty) and a minimally imperfect graph. 
Proof. Since $G$ is a properly fs-perfect graph, $G$ has a $(2 k+1)$-minimally imperfect node induced subgraph $G^{\prime}$. If $G^{\prime}=G$ the theorem follows. Otherwise, let $v \in V(G) \backslash V\left(G^{\prime}\right)$ and let $G_{v}$ be the subgraph of $G$ induced by $\{v\} \cup V\left(G^{\prime}\right)$. Clearly, $G_{v}$ is properly fs-perfect as well as LS + -perfect. Then, by Theorem 20 and Theorem 24, $G_{v} \notin \mathcal{F}^{k}$. So, $\delta_{G_{v}}(v)=2 k+1$ and $G_{v}=\{v\} \vee G^{\prime}$. Therefore, if $G^{\prime \prime}$ is the subgraph of $G$ induced by $V(G)-V\left(G^{\prime}\right), G=G^{\prime} \vee G^{\prime \prime}$. By Remark 9 $G^{\prime \prime}$ is a complete graph and by Remark 10 the result follows.

Since complete joins of complete graphs and minimally imperfect graphs are near-bipartite, they satisfy $\operatorname{NB}(G)=\operatorname{STAB}(G)$. Therefore, based on the results obtained so far, we can conclude that Conjecture 8 holds for fs-perfect graphs.

\section{Results CONCERNing THE $\mathrm{LS}_{+}$-OPERATOR}

In this section we include the proofs of some results on the $\mathrm{LS}_{+}$-operator that were stated without proof in the previous sections.

5.1. The $\mathbf{L S}_{+}$-imperfection of the graph $H^{k}$. Recall that $V\left(H^{k}\right)=\{0,1, \ldots, 2 k+1\}, H^{k}-$ $0=\overline{C_{2 k+1}}$ and $\delta(0)=2 k$. Without loss of generality, we may assume that the node $2 k+$ 1 in $H^{k}$ is the only one not connected with node 0 . Let us introduce the point $x(k, \gamma)=$ $\frac{1}{2 k+2+\gamma}(2,2, \ldots, 2,4)^{\top} \in \mathbb{R}^{2 k+2}$ where for $i \in\{1, \ldots, 2 k+2\}$, the $i$-th component of $x(k, \gamma)$ corresponds to the node $i-1$ in $H^{k}$, In what follows we show that $x(k, \gamma) \in \operatorname{LS}_{+}\left(H^{k}\right) \backslash \operatorname{STAB}\left(H^{k}\right)$ for some $\gamma \in(0,1)$ thus proving Theorem 18. We first consider $\beta_{k}=\frac{1}{2 k+2}, \gamma \in(0,1)$ and the $(2 k+3) \times(2 k+3)$ matrix given by

$$
Y(k, \gamma):=\left[\begin{array}{ccccccccc}
(2 k+2+\gamma) & 2 & 2 & 2 & 2 & 2 & \cdots & 2 & 4 \\
2 & 2 & 0 & 0 & 0 & 0 & \cdots & 0 & 2 \\
2 & 0 & 2 & 1-\beta_{k} & 0 & 0 & \cdots & 0 & 1+\beta_{k} \\
2 & 0 & 1-\beta_{k} & 2 & 1+\beta_{k} & 0 & \cdots & 0 & 0 \\
2 & 0 & 0 & 1+\beta_{k} & 2 & 1-\beta_{k} & \cdots & 0 & 0 \\
2 & 0 & 0 & 0 & 1-\beta_{k} & 2 & \cdots & 0 & 0 \\
\vdots & \vdots & \vdots & \vdots & \vdots & \vdots & \ddots & \vdots & \vdots \\
2 & 0 & 0 & 0 & 0 & 0 & \cdots & 2 & 1+\beta_{k} \\
4 & 2 & 1+\beta_{k} & 0 & 0 & 0 & \cdots & 1+\beta_{k} & 4
\end{array}\right] .
$$

Lemma 26. For $k \geq 3$, there exists $\gamma \in(0,1)$ such that $Y(k, \gamma)$ is PSD.

Proof. Let us denote by $\tilde{Y}(k)$ the $(2 k+2) \times(2 k+2)$ submatrix of $Y(k, \gamma)$ obtained after deleting the first row and column. Also consider $\hat{Y}(k)$, the Schur Complement of the $(1,1)$ entry of $\tilde{Y}(k)$, 
then

$$
\hat{Y}(k)=\left[\begin{array}{ccccccc}
2 & 1-\beta_{k} & 0 & 0 & \cdots & 0 & 1+\beta_{k} \\
1-\beta_{k} & 2 & 1+\beta_{k} & 0 & \cdots & 0 & 0 \\
0 & 1+\beta_{k} & 2 & 1-\beta_{k} & \cdots & 0 & 0 \\
0 & 0 & 1-\beta_{k} & 2 & \cdots & 0 & 0 \\
\vdots & \vdots & \vdots & \vdots & \ddots & \vdots & \vdots \\
0 & 0 & 0 & 0 & \cdots & 2 & 1+\beta_{k} \\
1+\beta_{k} & 0 & 0 & 0 & \cdots & 1+\beta_{k} & 2
\end{array}\right] .
$$

Claim 27. For every $k \geq 2, \tilde{Y}(k)$ is positive definite.

Proof. Let us first show that $\hat{Y}(k)$ is positive definite. For this purpose, we only need to verify that every leading principle minor of $\hat{Y}(k)$ is positive. Let us define $A_{0}(k):=1, B_{0}(k):=2$ and for $\ell \geq 1$,

$$
A_{\ell}(k):=\operatorname{det}\left[\begin{array}{ccccccc}
2 & 1-\beta_{k} & 0 & 0 & \cdots & 0 & 0 \\
1-\beta_{k} & 2 & 1+\beta_{k} & 0 & \cdots & 0 & 0 \\
0 & 1+\beta_{k} & 2 & 1-\beta_{k} & \cdots & 0 & 0 \\
0 & 0 & 1-\beta_{k} & 2 & \cdots & 0 & 0 \\
\vdots & \vdots & \vdots & \vdots & \ddots & \vdots & \vdots \\
0 & 0 & 0 & 0 & \cdots & 2 & 1-\beta_{k} \\
0 & 0 & 0 & 0 & \cdots & 1-\beta_{k} & 2
\end{array}\right] \text {, }
$$

where the matrix in the definition is $2 \ell \times 2 \ell$ and

$$
B_{\ell}(k)=\operatorname{det}\left[\begin{array}{ccccccc}
2 & 1-\beta_{k} & 0 & 0 & \cdots & 0 & 0 \\
1-\beta_{k} & 2 & 1+\beta_{k} & 0 & \cdots & 0 & 0 \\
0 & 1+\beta_{k} & 2 & 1-\beta_{k} & \cdots & 0 & 0 \\
0 & 0 & 1-\beta_{k} & 2 & \cdots & 0 & 0 \\
\vdots & \vdots & \vdots & \vdots & \ddots & \vdots & \vdots \\
0 & 0 & 0 & 0 & \cdots & 2 & 1+\beta_{k} \\
0 & 0 & 0 & 0 & \cdots & 1+\beta_{k} & 2
\end{array}\right] \text {, }
$$

where the matrix in the definition is $(2 \ell+1) \times(2 \ell+1)$. Using the determinant expansion on $A_{\ell}(k)$ and $B_{\ell}(k)$ we have that for every $\ell \geq 1$,

$$
\begin{gathered}
A_{\ell}(k)=2 B_{\ell-1}(k)-\left(1-\beta_{k}\right)^{2} A_{\ell-1}(k), \\
B_{\ell}(k)=2 A_{\ell}(k)-\left(1+\beta_{k}\right)^{2} B_{\ell-1}(k),
\end{gathered}
$$

and

$$
\begin{aligned}
\operatorname{det}(\hat{Y}(k)) & =2\left[A_{k}(k)-\left(1+\beta_{k}\right)^{2} B_{k-1}(k)+\left(1-\beta_{k}\right)^{k}\left(1+\beta_{k}\right)^{k+1}\right] \\
& =2\left[B_{k}(k)-A_{k}(k)+\left(1-\beta_{k}\right)^{k}\left(1+\beta_{k}\right)^{k+1}\right] .
\end{aligned}
$$


Using these recursive formulas, we have that $\hat{Y}(k)$ is positive definite. Finally, after the Schur Complement Lemma we have that $\tilde{Y}(k)$ is positive definite.

Using this claim we have:

Claim 28. Let $u$ be the (unique) vector such that

$$
\tilde{Y}(k) u=2\left(\mathbf{1}+\mathbf{e}_{2 k+2}\right) .
$$

Then $Y(k, \gamma)$ is PSD if and only if $\gamma \geq 1-\beta_{k} u_{2 k+2}$.

Proof. Using the Schur Complement Lemma for $\tilde{Y}(k)$ we have that $Y(k, \gamma)$ is PSD if and only if

$$
\tilde{Y}(k)-\frac{4}{2 k+2+\gamma}\left(\mathbf{1}+\mathbf{e}_{2 k+2}\right)\left(\mathbf{1}+\mathbf{e}_{2 k+2}\right)^{\top} \text { is PSD. }
$$

Using the automorphism $[\tilde{Y}(k)]^{-1 / 2} \cdot[\tilde{Y}(k)]^{-1 / 2}$ of the PSD cone, the latter is true if and only if the following matrix

$$
I-\frac{4}{2 k+2+\gamma}[\tilde{Y}(k)]^{-1 / 2}\left(\mathbf{1}+\mathbf{e}_{2 k+2}\right)\left(\mathbf{1}+\mathbf{e}_{2 k+2}\right)^{\top}[\tilde{Y}(k)]^{-1 / 2}
$$

is PSD. Since

$$
\frac{4}{2 k+2+\gamma}[\tilde{Y}(k)]^{-1 / 2}\left(\mathbf{1}+\mathbf{e}_{2 k+2}\right)\left(\mathbf{1}+\mathbf{e}_{2 k+2}\right)^{\top}[\tilde{Y}(k)]^{-1 / 2}
$$

is a rank one matrix, using (7) we have that the matrix in (8) is PSD if and only if

$$
1 \geq \frac{4}{2 k+2+\gamma}\left(\mathbf{1}+\mathbf{e}_{2 k+2}\right)^{\top}[\tilde{Y}(k)]^{-1}\left(\mathbf{1}+\mathbf{e}_{2 k+2}\right)=\frac{2\left(\mathbf{1}+\mathbf{e}_{2 k+2}\right)^{\top} u}{2 k+2+\gamma} .
$$

Now, using the definition of $\tilde{Y}(k)$ we have that

$$
\tilde{Y}(k) \mathbf{1}=4(\mathbf{1})+\left(4+2 \beta_{k}\right) \mathbf{e}_{2 k+2}=2 \tilde{Y}(k) u+2 \beta_{k} \mathbf{e}_{2 k+2},
$$

and then

$$
u=\frac{1}{2} \mathbf{1}-\beta_{k}[\tilde{Y}(k)]^{-1} \mathbf{e}_{2 k+2} .
$$

Therefore,

$$
\begin{aligned}
2\left(\mathbf{1}+\mathbf{e}_{2 k+2}\right)^{\top} u & =2\left(\mathbf{1}+\mathbf{e}_{2 k+2}\right)^{\top}\left(\frac{1}{2} \mathbf{1}-\beta_{k}[\tilde{Y}(k)]^{-1} \mathbf{e}_{2 k+2}\right) \\
& =(2 k+3)-2 \beta_{k}\left(\mathbf{1}+\mathbf{e}_{2 k+2}\right)^{\top}[\tilde{Y}(k)]^{-1} \mathbf{e}_{2 k+2}
\end{aligned}
$$

and again using (7), we obtain

$$
2\left(\mathbf{1}+\mathbf{e}_{2 k+2}\right)^{\top} u=(2 k+3)-\beta_{k} u_{2 k+2} .
$$

Hence, using (9) and $(10)$ we can conclude that the matrix in $(8)$ is PSD if and only if

$$
1 \geq \frac{(2 k+3)-\beta_{k} u_{2 k+2}}{2 k+2+\gamma}
$$

or equivalently, if and only if

$$
\gamma \geq 1-\beta_{k} u_{2 k+2}
$$


By the previous claims, to prove that $Y(k, \gamma)$ is PSD for some $\gamma \in(0,1)$, it suffices to prove that there exists $\gamma \in(0,1)$ such that

$$
\gamma \geq 1-\beta_{k} u_{2 k+2}
$$

where $u$ is the unique solution of (7). Thus, as long as $u_{2 k+2}>0$, we may have $\gamma<1$ as desired. Using (10) we have that

$$
u_{2 k+2}=\mathbf{e}_{2 k+2}^{\top} u=\frac{1}{2}-\beta_{k} \mathbf{e}_{2 k+2}^{\top} \tilde{Y}(k)^{-1} \mathbf{e}_{2 k+2}
$$

and by Cramer's rule and the definitions of $\tilde{Y}, \hat{Y}$, and $A_{k}$, we conclude

$$
u_{2 k+2}=\frac{1}{2}-\beta_{k} \frac{A_{k}}{\operatorname{det}(\hat{Y}(k))} .
$$

Then, using the recursive formula (6) we have that $u_{2 k+2}>0$. This completes the proof.

Utilizing the previous lemma we are able to prove Theorem 18 ,

Proof of Theorem 18 . Recall that we may assume that in $H^{k}$ the node $2 k+1$ is not connected with node 0 . Let $\gamma \in(0,1)$ and $x(k, \gamma)=\frac{1}{2 k+2+\gamma}(2,2, \ldots, 2,4)^{\top} \in \mathbb{R}^{2 k+2}$ where the $i$-th component of $x(k, \gamma)$ corresponds to node $i-1$ in $H^{k}$ for $i \in\{1, \ldots, 2 k+2\}$. Let $Y^{*}(k, \gamma)=$ $\frac{1}{2 k+2+\gamma} Y(k, \gamma)$. Then, $Y^{*}(k, \gamma)$ is a symmetric matrix that clearly satisfies that $Y^{*}(k, \gamma) \mathbf{e}_{0}=$ $\operatorname{diag}\left(Y^{*}(k, \gamma)\right) \in \operatorname{FRAC}\left(H^{k}\right)$. Moreover, it is not hard to check that, for $i \in\{1, \ldots, 2 k+2\}$,

$$
Y^{*}(k, \gamma) \mathbf{e}_{i} \in \operatorname{cone}\left(\operatorname{FRAC}\left(H^{k}\right)\right) \text { and } Y^{*}(k, \gamma)\left(\mathbf{e}_{0}-\mathbf{e}_{i}\right) \in \operatorname{cone}\left(\operatorname{FRAC}\left(H^{k}\right)\right) .
$$

This proves that $Y^{*}(k, \gamma) \in M\left(H^{k}\right)$. By the previous lemma, there exists $\bar{\gamma} \in(0,1)$ for which $Y^{*}(k, \bar{\gamma}) \in M_{+}\left(H^{k}\right)$. Hence, $x(k, \bar{\gamma}) \in \mathrm{LS}_{+}\left(H^{k}\right)$. It only remains to observe that $x(k, \bar{\gamma})$ violates the rank inequality of $H^{k}$. Thus, $x(k, \bar{\gamma}) \notin \operatorname{STAB}\left(H^{k}\right)$.

5.2. Operations that preserve $\mathbf{L S}_{+}$-imperfection. Firstly, we prove Theorem 22 on the $k$-stretching operation for $k \geq 1$, already stated in Section 4 . Actually, we will see that the same proof given in [26] for the case $k=0$ can be used for the case $k \geq 1$. Assume that $\tilde{G}$ is obtained from $G$ after the $k$-stretching operation on node $v$ and let $u, v_{1}$ and $v_{2}$ be as in the definition of the operation in Section 2.5 . For any $x \in \mathbb{R}^{V(G)}$, we write $x=\left(\begin{array}{c}\bar{x} \\ x_{v}\end{array}\right)$ where $\bar{x} \in \mathbb{R}^{V(G-v)}$.

For the case $k=0$, the authors in [26] prove that if a point $x=\left(\begin{array}{c}\bar{x} \\ x_{v}\end{array}\right) \in \operatorname{LS}_{+}^{r}(G)$ then the point $\tilde{x}$ given by

$$
x_{w}= \begin{cases}\tilde{x}_{w} & \text { if } w \in\left\{u, v_{1}, v_{2}\right\} \\ \bar{x}_{w} & \text { otherwise }\end{cases}
$$

satisfies $\tilde{x} \in \operatorname{LS}_{+}^{r}(\tilde{G})$. In order to do so they prove that if

$$
Y=\left[\begin{array}{c|c|c}
1 & \bar{x}^{\top} & x_{v} \\
\hline \bar{x} & \bar{X} & \bar{y} \\
\hline x_{v} & \bar{y}^{\top} & x_{v}
\end{array}\right] \in M_{+}\left(\operatorname{LS}_{+}^{r-1}(G)\right)
$$


then

$\tilde{Y}=\left[\begin{array}{c|c|c|c|c}1 & \bar{x}^{\top} & x_{v} & x_{v} & \left(1-x_{v}\right) \\ \hline \bar{x} & \bar{X} & \bar{y} & \bar{y} & \bar{x}-\bar{y} \\ \hline x_{v} & \bar{y}^{\top} & x_{v} & x_{v} & 0 \\ \hline x_{v} & \bar{y}^{\top} & x_{v} & x_{v} & 0 \\ \hline\left(1-x_{v}\right) & (\bar{x}-\bar{y})^{\top} & 0 & 0 & \left(1-x_{v}\right)\end{array}\right] \in M_{+}\left(\operatorname{LS}_{+}^{r-1}(\tilde{G})\right)$.

On the other hand, they show that if $\sum_{j \in V(G)} a_{j} x_{j} \leq \beta$ is a valid inequality for $\operatorname{STAB}(G)$, defining $\tilde{\beta}=\beta+a_{v}$ and

$$
\tilde{a}_{j}= \begin{cases}a_{v} & \text { if } j \in\left\{v_{1}, v_{2}, u\right\} \\ a_{j} & \text { otherwise }\end{cases}
$$

the inequality $\sum_{j \in V(\tilde{G})} \tilde{a}_{j} x_{j} \leq \tilde{\beta}$ is valid for $\operatorname{STAB}(\tilde{G})$. Moreover, if $x^{*}$ violates $\sum_{j \in V(G)} a_{j} x_{j} \leq$ $\beta$ then $\tilde{x}^{*}$ violates $\sum_{j \in V(\tilde{G})} \tilde{a}_{j} x_{j} \leq \tilde{\beta}$.

Proof of Theorem 22. It is enough to observe that $\tilde{Y} \in M_{+}\left(\operatorname{LS}^{r-1}(\tilde{G})\right)$ and the inequality $\sum_{j \in V(\tilde{G})} \tilde{a}_{j} x_{j} \leq \tilde{\beta}$ is valid for $\operatorname{STAB}(\tilde{G})$ even for the case $\tilde{G}$ is obtained after the $k$-stretching on node $v$ in $G$, for $k \geq 1$.

Let us now consider the clique-subdivision operation defined in [4]. For $x \in \mathbb{R}^{n}$, let $\bar{x} \in \mathbb{R}^{n+2}$ such that $\bar{x}_{i}=x_{i}$ for every $i \in\{1, \ldots, n\}, \bar{x}_{n+1}=x_{2}$ and $\bar{x}_{n+2}=x_{1}$, and write $\bar{x}=\left(\begin{array}{c}x \\ x_{2} \\ x_{1}\end{array}\right)$. In [4] the authors prove that if $\tilde{G}$ be obtained from $G$ by the clique subdivision of the edge $v_{1} v_{2}$ in the clique $K$ and $x \in \operatorname{LS}_{+}^{k}(G)$ then $\bar{x} \in \operatorname{LS}^{k}(\tilde{G})$. In order to do so, they show that if $Y \mathbf{e}_{0}=\left(\begin{array}{c}1 \\ x\end{array}\right)$ for

$Y=\left[\begin{array}{c|c|c|c}1 & x_{1} & x_{2} & \bar{x}^{\top} \\ \hline x_{1} & x_{1} & 0 & y_{1}^{\top} \\ \hline x_{2} & 0 & x_{2} & y_{2}^{\top} \\ \hline \tilde{x} & y_{1} & y_{2} & \bar{X}\end{array}\right] \in M\left(\operatorname{LS}^{r-1}(G)\right)$


then

$$
\tilde{Y}=\left[\begin{array}{c|c|c|c|c|c}
1 & x_{1} & x_{2} & \tilde{x}^{\top} & x_{2} & x_{1} \\
\hline x_{1} & x_{1} & 0 & y_{1}^{\top} & 0 & x_{1} \\
\hline x_{2} & 0 & x_{2} & y_{2}^{\top} & x_{2} & 0 \\
\hline \tilde{x} & y_{1} & y_{2} & \bar{X} & y_{2} & y_{1} \\
\hline x_{2} & 0 & x_{2} & y_{2}^{\top} & x_{2} & 0 \\
\hline x_{1} & x_{1} & 0 & y_{1}^{\top} & 0 & x_{1}
\end{array}\right] \in M\left(\operatorname{LS}^{r-1}(\tilde{G})\right) .
$$

Proof of Theorem 23. It is enough to observe that if the matrix $Y$ is PSD then so is the matrix in $(12)$.

\section{Conclusions And Further Results}

In this work, we face the problem of characterizing the stable set polytope of $\mathrm{LS}_{+}$-perfect graphs, a graph class where the Maximum Weight Stable Set Problem is polynomial time solvable. This class strictly includes many well-known graph classes such as perfect graphs, $t$-perfect graphs, wheels, anti-holes, near-bipartite graphs and the graphs obtained from various suitable compositions of these. The stable set polytope of either a perfect or a near-bipartite graph only needs the inequalities associated with the stable set polytopes of its near-bipartite subgraphs. In a previous work, we have conjectured that the same holds for all $\mathrm{LS}_{+}$-perfect graphs. In this paper, we prove the validity of this conjecture for fs-perfect graphs, a superclass of near-perfect graphs. Moreover, if $\mathcal{F S}$ denotes the class of fs-perfect graphs, using the definition in (1), we actually prove that the conjecture holds for a superclass of fs-perfect graph defined as those graphs for which $\mathcal{F S}(G)=\operatorname{STAB}(G)$. Observe that the graph in Figure 4 satisfies $\mathcal{F} \mathcal{S}(G)=\operatorname{STAB}(G)$ and it is not fs-perfect.

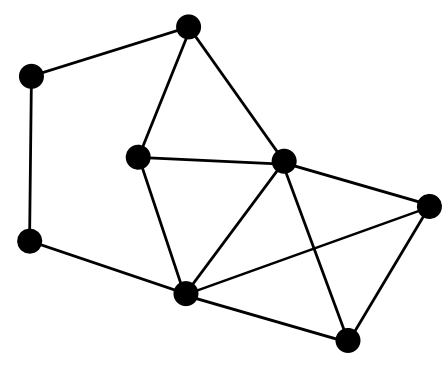

Figure 4. A graph $G$ satisfying $\mathcal{F S}(G)=\operatorname{STAB}(G)$ which is not fs-perfect.

Also, the results used in the proof of the Theorem 25 allow us to conclude the following:

Corollary 29. Let $G$ be a graph such that $V(G)=\{0,1, \ldots, 2 k+1\}$ with $k \geq 2$ and $G-0$ is minimally imperfect. Then:

- If $G-0=C_{2 k+1}$ then $G$ is $\mathrm{LS}_{+}$-perfect if and only if either $\delta_{G}(0) \geq 2$ and $G$ has only one odd central cycle or $\delta_{G}(0) \in\{0,1,2 k+1\}$. 
- If $G-0=\overline{C_{2 k+1}}$ with $\alpha(G)=2$ then $G$ is $\mathrm{LS}_{+}$-perfect if and only $\delta_{G}(0)=2 k+1$.

From the above characterization we identify the forbidden structures in the family of $\mathrm{LS}_{+^{-}}$ perfect graphs. In other words,

Corollary 30. Let $G$ be an $\mathrm{LS}_{+}$-perfect graph. Then, there is no subgraph $G^{\prime}$ of $G$ such that

- $G^{\prime}-v_{0}=C_{2 k+1}, 2 \leq \delta_{G^{\prime}}\left(v_{0}\right) \leq 2 k$ and $G^{\prime}$ has at least two odd central cycles, or

- $G^{\prime}-v_{0}=\overline{C_{2 k+1}}$ and $k+1 \leq \delta_{G}^{\prime}\left(v_{0}\right) \leq 2 k$ and $\alpha\left(G^{\prime}\right)=2$,

for some $v_{0} \in V\left(G^{\prime}\right)$ and $k \geq 2$.

\section{REFERENCES}

[1] Y. H. Au, A Comprehensive Analysis of Lift-and-Project Methods for Combinatorial Optimization, PhD Thesis, Department of Combinatorics and Optimization, Faculty of Mathematics, University of Waterloo, Canada, August 2014.

[2] Y. H. Au, On the Polyhedral Lift-and-Project Rank Conjecture for the Fractional Stable Set Polytope, M.Math. Thesis, Department of Combinatorics and Optimization, Faculty of Mathematics, University of Waterloo, Canada, January 2008.

[3] Y. H. Au and L. Tunçel, On the polyhedral lift-and-project methods and the fractional stable set polytope, Discrete Optimization 6 (2009) 206-213.

[4] N. Aguilera, M. Escalante and P. Fekete, On the facets of lift-and-project relaxations under graph operations. Discrete Applied Mathematics 164 (2014) 360-372.

[5] C. Berge, Perfect graphs, Six Papers on Graph Theory, Calcutta: Indian Statistical Institute, 1963, 1-21.

[6] S. Bianchi, M. Escalante, G. Nasini and L. Tunçel, Some advances on Lovász-Schrijver $N_{+}($.$) relaxations on$ the fractional stable set polytope, Electronic Notes in Discrete Mathematics 37 (2011) 189-194.

[7] S. Bianchi, M. Escalante, G. Nasini and L. Tunçel, Near-perfect graphs with polyhedral $N_{+}(G)$, Electronic Notes in Discrete Mathematics 37 (2011) 393-398.

[8] S. Bianchi, M. Escalante, G. Nasini and L. Tunçel, Lovász-Schrijver SDP-operator and a superclass of nearperfect graphs. Electronic Notes in Discrete Mathematics 44 (2013) 339-344.

[9] D. Bienstock and N. Ozbay, Tree-width and the Sherali-Adams operator, Discrete Optim. 1 (2004) 13-21.

[10] M. K. de Carli Silva, Geometric Ramifications of the Lovász Theta Function and Their Interplay with Duality, PhD Thesis, Department of Combinatorics and Optimization, Faculty of Mathematics, University of Waterloo, Canada, August 2013.

[11] M. Chudnovsky, N. Robertson, P. Seymour and R. Thomas, Progress on perfect graphs, Math. Program. 97 (2003) 405-422.

[12] M. Chudnovsky, G. Cornuéjols, X. Liu, P. Seymour and K. Vuskovic, Recognizing Berge Graphs, Combinatorica 25 (2005) 143-186.

[13] M. Chudnovsky, N. Robertson, P. Seymour, R. Thomas, The strong perfect graph theorem, Annals of Mathematics 164 (2006) 51-229.

[14] V. Chvátal, On certain polytopes associated with graphs, Journal of Combinatorial Theory B 18 (1975) 138-154.

[15] E. de Klerk and D. V. Pasechnik, Approximation of the stability number of a graph via copositive programming, SIAM J. Optim., 12 (2002) 875-892.

[16] M. Escalante, M.S. Montelar and G. Nasini, Minimal $N_{+}$-rank graphs: Progress on Lipták and Tunçel's conjecture, Oper. Res. Lett. 34 (2006) 639-646.

[17] M. Escalante and G. Nasini, Lovász and Schrijver $N_{+}$-relaxation on web graphs, manuscript 2014.

[18] M. Giandomenico, F. Rossi and S. Smriglio, Strong lift-and-project cutting planes for the stable set problem, Math. Program. 141 (2013) 165-192.

[19] M. Giandomenico, A. Letchford, F. Rossi and S. Smriglio, An application of the Lovász-Schrijver $M(K, K)$ operator to the stable set problem, Math. Program. 120 (2009) 381-401. 
[20] M. Grötschel, L. Lovász and A. Schrijver, The ellipsoid method and its consequences in combinatorial optimization, Combinatorica 1 (1981) 169-197.

[21] M. Grötschel, L. Lovász and A. Schrijver, Geometric Algorithms and Combinatorial Optimization, Springer, New York, 1988.

[22] N. Gvozdenović and M. Laurent, Semidefinite bounds for the stability number of a graph via sums of squares of polynomials, Math. Program. 110 (2007) 145-173.

[23] M. Laurent, A comparison of the Sherali-Adams, Lovász-Schrijver, and Lasserre relaxations for 0-1 programming, Math. Oper. Res. 28 (2003) 470-496.

[24] L. Lipták, Critical Facets of the Stable Set Polytope, Ph.D. Thesis, Yale University, U.S.A., 1999.

[25] L. Lipták and L. Lovász, Critical facets of the stable set polytope, Combinatorica 21 (2001) 61-88.

[26] L. Lipták and L. Tunçel, Stable set problem and the lift-and-project ranks of graphs, Math. Program. 98 (2003) 319-353.

[27] L. Lovász, On the Shannon capacity of a graph, IEEE Transactions 25 (1979) 1-7.

[28] L. Lovász and A. Schrijver, Cones of matrices and set-functions and 0-1 optimization, SIAM J. Optim. 1 (1991) 166-190.

[29] G. Nasini, Caracterización de los grafos fs-perfectos, Reunión Anual de Comunicaciones Científicas de la Unión Matemática Argentina, Tucumán, Argentina, 2011.

[30] M. Padberg, On the facial structure of set packing polyhedra, Math. Program. 5 (1973) 199-215.

[31] J. Peña, J. Vera and L. F. Zuluaga, Computing the stability number of a graph via linear and semidefinite programming, SIAM J. Optim. 18 (2007) 87-105.

[32] F. B. Shepherd, Near-Perfect matrices, Math. Program. 64 (1994) 295-323.

[33] F. B. Shepherd, Applying Lehman's theorems to packing problems, Math. Program. 71 (1995) 353-367.

[34] H. D. Sherali and Y. Lee, Tighter representations for set partitioning problems, Discrete Appl. Math. 68 (1996) 153-167.

[35] L. A. Wolsey, Further facet generating procedures for vertex packing polytopes, Math. Program. 11 (1976) 158-163. 